\title{
EDITORIAL SPECIAL ISSUE ON AGENT-BASED MODELLING FOR COMPLEX SYSTEMS
}

\author{
Quan Bai ${ }^{1} \quad$ Fenghui Ren ${ }^{2}$

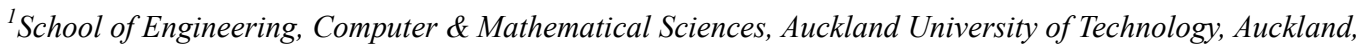 \\ New Zealand \\ quan.bai@aut.ac.nz (凹) \\ ${ }^{2}$ School of Computing and Information Technology, University of Wollongong, Wollongong, Australia
} fren@uow.edu.au

Computer based modelling and simulation has become useful tools to facilitate humans to understand systems in different domains, such as physics, astrophysics, chemistry, biology, economics, engineering and social science. A complex system is featured with a large number of interacting components (agents, processes, etc.), whose aggregate activities are nonlinear and self-organized. Complex systems are hard to be simulated or modelled by using traditional computational approaches due to the complex relationships of components and distributed features of resources, and dynamic work environments. Meanwhile, smart systems such as multi-agent systems have demonstrated advantages and great potentials in modelling and simulating complex systems.

This special issue aims to bring together researchers in both Artificial Intelligence and system modelling and simulation to discuss research challenges and cutting edge techniques in smart simulation and modelling of complex systems. The topics of this issue include but are not limited to the modelling and simulation of different complex systems such as agent-based systems, large-scale systems, dynamic environment and ecosystems, smart grid systems, social and economic organizations, market-based systems, transportation systems, and emergency management systems.

This special issue contains the selected extended papers presented at the 3rd International Workshop on Smart Simulation and Modelling for Complex System jointly organized with the 25th International Conference on Artificial Intelligence at Melbourne Australia in August 2017 as well as some new submissions. After the peerreview process, seven papers were accepted.

Le, Zhang and Ren (2018) proposed an economic broker-based matching approach between a buyer's requirements and a seller's offers in e-marketplaces which enable the broker to model the seller's price policy, the buyer's 
buying requirements, so as to match potential buyers and sellers in the e-marketplaces. The proposed approach can significantly increase the trading success rate in e-marketplaces and well balance the profits between the buyer, the seller and the broker.

Chen, Yu, Diao, Liu and Wang (2018) proposed an integrated solution to decision making between social learning and individual learning in multiagent systems. Two integration modes have been proposed that enable agents to choose in between these two learning strategies, either in a fixed or in an adaptive manner. Experimental evaluations have shown that these two kinds of leaning strategies have different roles in maintaining efficient coordination among agents. These differences can reveal some significant insights into the manipulation and control of agent behaviors in multiagent systems, and also shed light on understanding the social factors in shaping coordinated behaviors in humans and animals.

Kakimoto and Fujita (2018) proposed an effective agent-based negotiation approach to perform automated negotiations with nonlinear issues. The proposed approach was based on recursive partitioning using an issue dendrogram. A mediator first finds partial agreements in each sub-space based on bids from agents, then combines them to produce the final agreement. When a solution can't be found, the proposed approach can recursively decompose the negotiation sub-problems using the issue dendrogram. It is demonstrated that the proposed approach can generate higher-optimality outcomes with greater scalability than the state-of-art work.

$\mathrm{Su}$, Wang, Jia, Guo and Ding (2018) proposed the two multi-objective coalition formation models to handle the task allocation in disaster rescue domain by considering the dynamic environment, the heterogeneity and complexity of rescue tasks. First, through coalition formation, the proposed models enable agents to cooperatively perform complex tasks that cannot be completed by single agent. In addition, through adjusting the weights of multiple task allocation objectives, the proposed models can employ the linear programming to generate more adaptive task allocation plans, which can satisfy different task allocation requirements in disaster rescue. Finally, through employing the multi-stage task allocation mechanism of the dynamic programming, the proposed models can handle the dynamics of tasks and agents in disaster environments. Experimental results indicate that the proposed models have good performance on coalition formation for task allocation in disaster environments

$\mathrm{Xu}$, Yang, Li and Huang (2018) proposed a new method to improve the intelligence of virtual entities in Computer Generated Forces (CGFs) construction by using Monte Carlo Tree Search (MCTS). The work formulated the tree model through the state and action abstraction, 
and extend its expansion process to handle simultaneous and durative moves. The Hierarchical Task Network (HTN) planning was also employed to guide the search, thus enhancing the search efficiency. The final implementation is tested in an infantry combat simulation where a company commander needs to control three platoons to assault and clear enemies within defined areas. Comparative results from a series of experiments demonstrate that the HTN guided MCTS commander can outperform other commanders following fixed strategies.

Wu, Bai and Sengvong (2018) introduced the GreenCommute, a novel recommendation system which can facilitate commuters to take public friendly commute options, while provide support to alleviate the external cost in society, such as traffic pollution, congestion and accidents. In the meanwhile, a rewarding mechanism for persuading commuters is embedded in the proposed approach for balancing the conflict between personal needs and social aims. The allocation of reward values also takes users' influential degrees in the social network into consideration. Experimental results show that the GreenCommute can promote public friendly commute options more effectively in comparison to the traditional recommendation system.

Aydogan, Marsa-Maestre, Klein and Jonker (2018) pursued the problem of selecting the effective negotiation mechanism by (1) defining a set of scenario metrics to capture the relevant features of negotiation problems, (2) evaluating the performance of a range of negotiation mechanisms on a diverse test suite of negotiation scenarios, (3) applying machine learning techniques to identify which mechanisms work best with which scenarios, and (4) demonstrating that using these classification rules for mechanism selection enables significantly better negotiation performance than any single mechanism alone. It is believed that the proposed approach can help agent to select the most suitable negotiation mechanism based on the specific negotiation setting.

\section{References}

[1] Aydoğan, R., Marsa-Maestre, I., Klein, M. \& Jonker, C.M. (2018). A machine learning approach for mechanism selection in complex negotiations. Journal of Systems Science and Systems Engineering, 27(2):134-155.

[2] Chen, B.C., Yu, C., Diao, Q.S., Liu, R. \& Wang, Y.L.(2018). Social or individual learning? An aggregated solution for coordination in multiagent systems. Journal of Systems Science and Systems Engineering, 27(2):180-200.

[3] Kakimoto, S. \& Fujita, K.(2018). Effective automated negotiation based on issue dendrograms and partial agreements. Journal of Systems Science and Systems Engineering, 27(2):201-214.

[4] Le, D.T., Zhang, M.J. \& Ren, F.H. (2018). 
An economic model-based matching approach between buyers and sellers through a broker in an open e-marketplace. Journal of Systems Science and Systems Engineering, 27(2):156-179.

[5] Su, X., Wang, Y.C., Jia, X.B., Guo, L.M. \& Ding, Z.M. (2018). Two innovative coalition formation models for dynamic task allocation in disaster rescues. Journal of Systems Science and Systems Engineering, 27(2):215-230.

[6] Wu, S.Q., Bai, Q. \& Sengvong, S. (2018). Greencommute: an influence-aware persuasive recommendation approach for public-friendly commute options. Journal of Systems Science and Systems Engineering, 27(2):250-264.

[7] Xu, X., Yang, M. \& Li, G. (2018). Adaptive CGF commander behavior modeling through HTN guided Monte Carlo tree search. Journal of Systems Science and Systems Engineering, 27(2):231-249.

Quan Bai is a senior lecturer at the School of Computing and Mathematical Sciences, Auckland University of Technology, New Zealand. Dr Quan Bai received his PhD (2007) and MSc (2002) from the University of Wollongong, Australia, and graduated with double bachelor's degree (2002) from Tianjin
University, China. After he received his $\mathrm{PhD}$, Quan worked as a postdoctoral research follow for the University of Wollongong (2007-2009), and for the Commonwealth Scientific and Industrial Research Organization (CSIRO) (2009-2011). Quan's research interests are mainly focused on intelligent systems, knowledge discovery and crowd intelligence. He has published more than 90 papers in related fields.

Fenghui Ren is a lecturer at the School of Computing and Information Technology, the University of Wollongong, Australia. $\mathrm{He}$ received his $\mathrm{PhD}$ (2010) from the University of Wollongong, Australia. Between 2010 and 2016, he worked on several projects related to agent-based complex systems modelling and simulation in the University of Wollongong as an ARC postdoctoral fellow, an Vice Chancellor's Postdoctoral Fellow, and an ARC DECRA Fellow, respectively. From 2017, he became a formal lecturer. His research interests include intelligent systems, agent and multi-agent systems, data mining and knowledge discovery, and smart grid. Dr. Ren is an active researcher and published more than 50 papers. 